\title{
New Types of Cryptanalytic Attacks Using Related Keys
}

\author{
Eli Biham ${ }^{1}$ \\ Computer Science Department \\ Technion - Israel Institute of Technology \\ Haifa 32000, Israel
}

\begin{abstract}
In this paper we study the influence of key scheduling algorithms on the strength of blockciphers. We show that the key scheduling algorithms of many blockciphers inherit obvious relationships between keys, and use these key relations to attack the blockciphers. Two new types of attacks are described: New chosen plaintext reductions of the complexity of exhaustive search attacks (and the faster varjants based on complementation properties), and new low-complexity chosen key attacks. These attacks are independent of the number of rounds of the cryptosystems and of the details of the $F$-function and may have very small complexities. These attacks show that the key scheduling algorithm should be carefully designed and that its structure should not be too simple. These attacks are applicable to both variants of LOKI and to Lucifer, DES is not vulnerable to the related keys attacks since the shift pattern in the key scheduling algorithm is not the same in all the rounds.
\end{abstract}

\section{Introduction}

In this paper we describe new types of attacks on blockciphers: chosen plaintext reductions of the complexity of exhaustive search and chosen key attacks in which only the relations between pairs of related keys are chosen by the attacker, who does not know the keys themselves. The chosen plaintext attacks reduce the complexity of exhaustive search and the complexity of the faster chosen plaintext attacks based on complementation properties by a factor of three. The chosen key attacks have very low complexities, however, they can be used only whenever the attacker can choose the relationships between unknown keys and wish to know the keys themselves.

These attacks are based on the observation that in many blockciphers we can view the key scheduling algorithm as a set of algorithms, each of which extracts one particular subkey from the subkeys of the previous few rounds. If all the algorithms of extracting the subkeys of the various rounds are the same, then given a key we can shift all the subkeys one round backwards and get a new set of valid subkeys which can be derived from some other key. We call these keys related keys.

An interesting feature of the attacks based on related keys is that they are independent of the number of rounds of the attacked cryptosystem. These attacks are applicable to both rariants of LOKI[5.t]. and to Lucifer[16]. Nevertheless. they are not applicable to DES[14]

\footnotetext{
Acknowledgment: This research was supported by the fund for the promotion of research at the Teclinion.
} 
due to the observation that the number of shifts of the key registers $(C$ and $D)$ in the key scheduling algorithm is not the same in all the rounds. However, if the shifts by one bit in the key scheduling of DES would be replaced by shifts by two bits. DES would become vulnerable to this kind of attack as well.

Another potential application of related keys is to analyze hash functions (either hash functions based on blockciphers or general hash functions). It may be possible in such functions to choose the message in a way that the related keys property suggest an additional message with the same hash value. Currently, we are not aware of a particular such application to hash functions. but designers of hash functions should be careful to design their functions immune to this weakness.

The results of the attacks are as follows: The complexity of a chosen plaintext attack on LOKI89 is about $1.5 \cdot 2^{54}$, which is almost three times faster than previously reported chosen plaintext attacks. The chosen key chosen plaintext attack takes a few seconds on a personal computer and its complexity is about $2^{17}$, and the complexity of the chosen key known plaintext attack is about $2^{32}$. The corresponding complexities of the attacks on the newer LOKI91 are $1.375 \cdot 2^{61}, 2^{32}$, and $2^{48}$ respectively. The complexity of the chosen key chosen plaintext attack on Lucifer is about $2^{33}$. The DES, the IDEA cipher[11,12] and the FEAL cipher[15,13] are not vulnerable to these attacks.

Recently, Lars Ramkilde Knudsen found independently[9] the basic concept of the chosen plaintext related keys attacks and applied it to LOK191. However, his attack (whose complexity is $1.07 \cdot 2^{62}$ ) is still $50 \%$ slower than the corresponding attack we present in this paper.

\section{Description of LOKI89 and LOKI91}

LOKI is a family of blockciphers with two variants: The original LOKI cipher, which was renamed to LOKI89[5], and the newer variant LOKI91[4]. Both variants have a structure similar to DES[14], with replaced $F$ function and initial and final permutations and a replaced key scheduling algorithm. The new $F$ function XORs the right half of the data with the subkey and expands the result to 48 bits, which enter into four 12-bit to 8-bit $\mathrm{S}$ boxes. The output of the $S$ boxes is concatenated and permuted to form the output of the $F$ function. In LOKI89 (see Figure 1), the initial and the final permutations are replaced by transformations which exclusive-or the data with the key. The key scheduling algorithm takes a 64 -bit key, declares its left half as the value of $\mathrm{K} 1$ and its right half as the value of K2. Each other subkey $\mathrm{K} i$ (out of $\mathrm{K} 3, \ldots, \mathrm{K} 16$ ) is defined by rotating the subkey $\mathrm{K} j$ of round $j=i-2$ by 12 bits to the left $(\mathrm{K} i=\mathrm{ROL12}(\mathrm{K} j))$. Thus, all the subkeys of the odd rounds share the same bits and all the subkeys of the even rounds share the same bits.

LOKI91 (see Figure 2) differs from LOKI89 by the choice of the S boxes, which are chosen to hold better against differential cryptanalysis. The initial and the final permutations are eliminated. The new key scheduling algorithm declares the value of the left half of the key to be $\mathrm{KI}$ and the same value rotated 12 bits to the left is declared to be $\mathrm{K} 2$. The value of the right half of the key is declared to be $\mathrm{K} 3$ and the same value rotated 12 bits to the left is declared as $\mathrm{K} 4$. Each other subkey $\mathrm{K} i$ (out of $\mathrm{K} 5, \ldots, \mathrm{K} 16$ ) is defined by rotating the subkey $K j$ of round $j=i-4$ by 25 bits to the left ( $K i=\operatorname{ROL25}(\mathrm{K} j)$ ). Still, the subkeys share bits with a very structured order. 


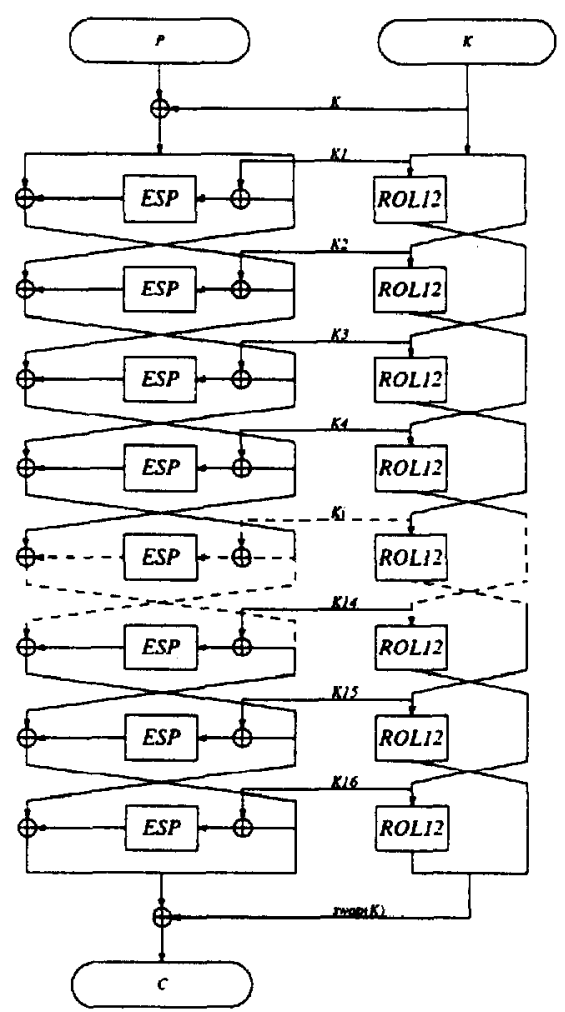

Figure 1. Outline of LOKI89.

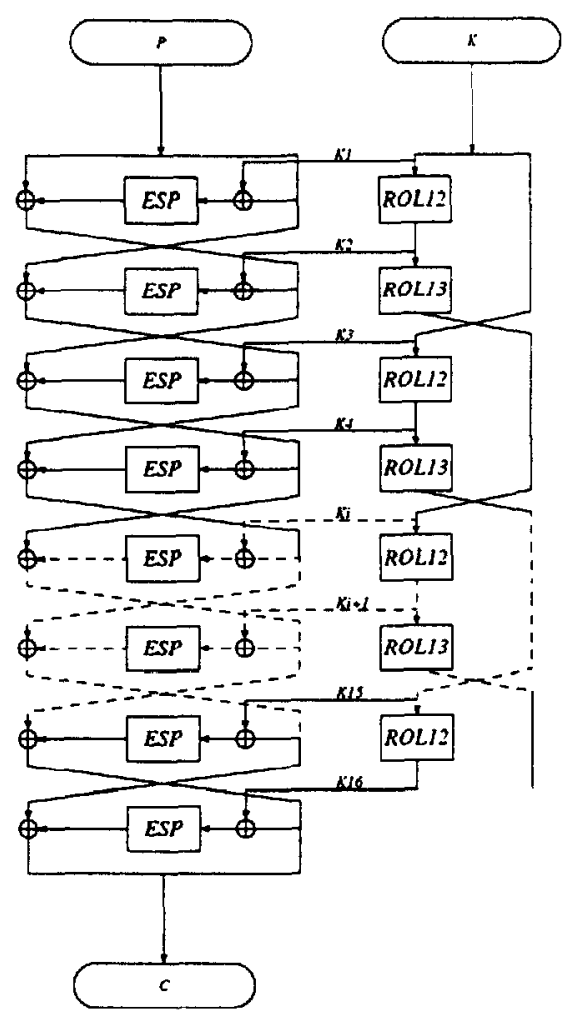

Figure 2. Outline of LOKI91.

\section{The Chosen Key Attacks}

In the chosen key attacks, two related keys with certain relationship are used and several plaintexts are encrypted under each of them. The attacker knows only the relationship between the two keys, but not the keys themselves. He receives the ciphertexts and use them to find both keys. Two kinds of chosen key attacks are studied: a chosen key known plaintext attack in which only the relation between the keys is chosen by the attacker, and a chosen key chosen plaintext attack in which the attacker chooses the relation between the keys as well as the plaintexts to be encrypted. These attacks are independent of the exact number of rounds of the attacked cryptosystem, and even if the number of rounds is enlarged (and especially if doubled), the resulting cryptosystem remains vulnerable to the same attack.

\section{$3.1 \quad$ LOKI89}

In LOKIS9. every choice of two subkeys, one from an odd round and one from an even round. have a corresponding 64 -bit key. Since all the algorithms of deriving the subkeys from the two preceding subkeys are the same, the position of the rounds in which two subleys present does not affect the derivation of the following subkeys (nor the preceding ones). If we only fix two subkeys $K 2$ and $K 3$ of a key $K$, and define a second key $K^{*}$ by choosing $K^{-}=K_{2} 2$ and 


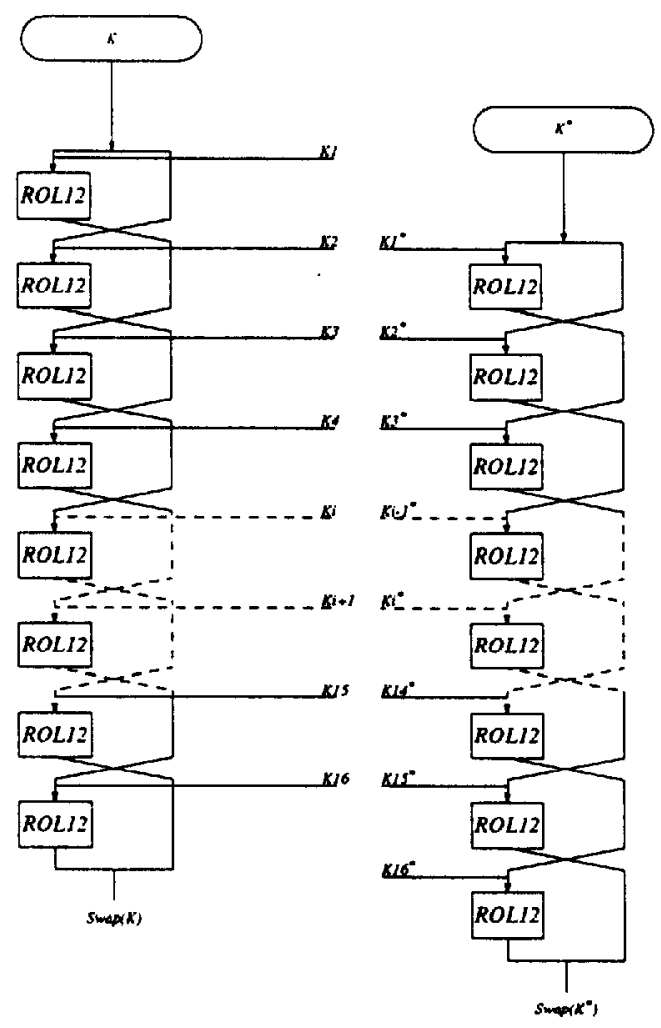

Figure 3. Relations of subkeys in the key scheduling algorithm of LOKIS9.

$K 2^{*}=\mathrm{K} 3$. then the values of the subkeys $K i^{*}$ of the key $\mathrm{K}^{*}$ are the same as of the following subkeys $\mathrm{K}(i+1)$ of the key $\mathrm{K}$. In this case, $\mathrm{K}^{*}=(\mathrm{K} 2, \mathrm{~K} 3)=\left(\mathrm{K}_{R}, \mathrm{ROL12}\left(\mathrm{K}_{L}\right)\right)$. Therefore, the following property holds for any two such related keys: If the data before the second round in an encryption under the key $\mathrm{K}$ equals the data before the first round in an encryption under the key $\mathrm{K}^{-}$. then the data and the inputs to the $F$ functions are the same in both executions with a difference of one round. In this case, if the plaintext $P$ is encrypted under the key $\mathrm{K}$, then the data before the second round is $\left(P_{R} \oplus \mathrm{K}_{R}, P_{L} \oplus \mathrm{K}_{L} \oplus F\left(P_{R} \oplus \mathrm{K}_{R}, \mathrm{~K}_{L}\right)\right)$. This data equals the data before the first round in the other encryption under the key $\mathrm{K}^{*}$, whose value is $P^{*} \oplus \mathrm{K}^{*}=\left(P_{L}^{*} \oplus \mathrm{K}_{R}, P_{R}^{*} \oplus \mathrm{ROL} 12\left(\mathrm{~K}_{L}\right)\right)$, and thus in such a pair

$$
P^{*}=\left(P_{R}, P_{L} \oplus \mathrm{K}_{L} \oplus \operatorname{ROL} 12\left(\mathrm{~K}_{L}\right) \oplus F\left(P_{R} \in \mathrm{K}_{R}, \mathrm{~K}_{L}\right)\right) \text {. }
$$

We see that the right half of $P$ equals the left half of $P^{*}$ and that the relation between the other halves depends on the keys. In such a pair. there is also a similar a relation between the ciphertexts

$$
C^{*}=\left(C_{R} \oplus \mathrm{K}_{L} \oplus \mathrm{ROL} 12\left(\mathrm{~K}_{L}\right) \oplus F\left(C_{L} \oplus \mathrm{K}_{R}, \mathrm{~K}_{L}\right), C_{L}\right)
$$

Figures 3 and 4 describes the relations between the subkeys of the two keys and the relations between the values during the two encryptions.

A chosen key chosen plaintext attack based on this property chooses a 32-bit value $P_{R}$, $2^{16}$ plaintexts $P_{0} \ldots, P_{65535}$ whose right halves equal $P_{R}$ and whose 32 -bit left halves are 


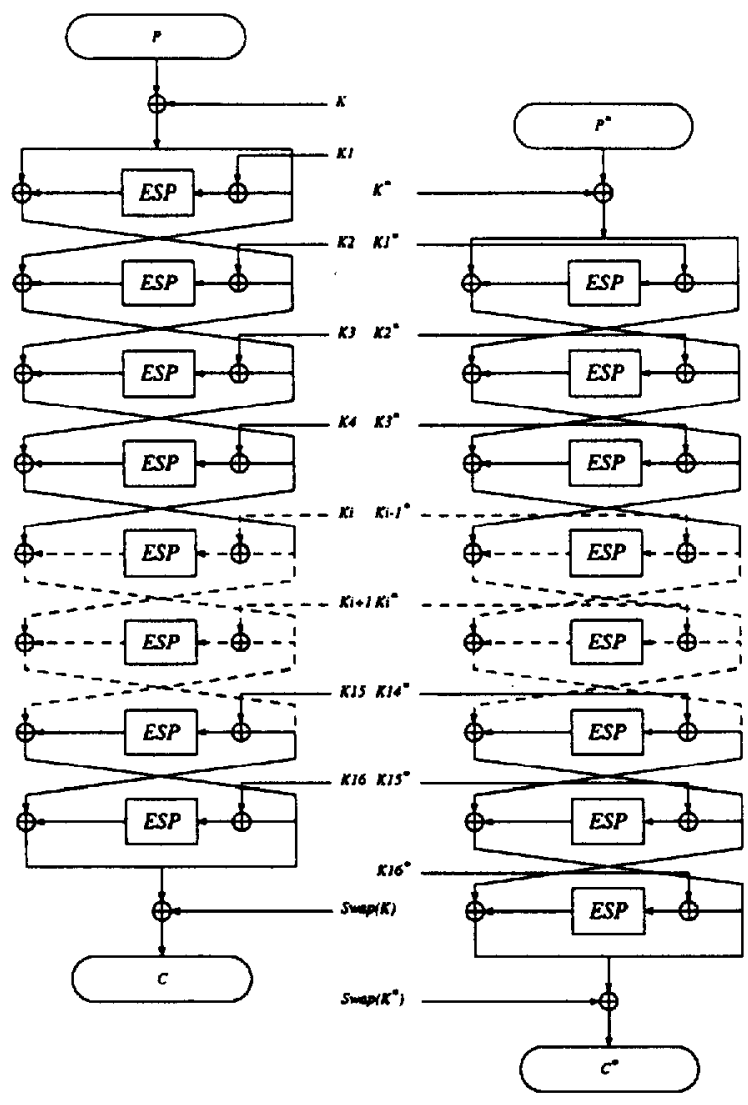

Figure 4. Relations during LOKI89 encryption.

randomly chosen, and $2^{16}$ plaintexts $P_{0}^{*} \ldots, P_{65535}^{*}$ whose left halves equal $P_{R}$ and whose 32-bit right halves are randomly chosen. Two unknown related keys are used to encrypt these plaintexts on the target machine: a key $\mathrm{K}$ is used to encrypt the first $2^{16}$ plaintexts and the key $\mathrm{K}^{*}=\left(\mathrm{K}_{R}, \mathrm{ROL1} 2\left(\mathrm{~K}_{L}\right)\right)$ is used to encrypt the other $2^{16}$ plaintexts. In every pair of plaintexts $P_{i}$ and $P_{j}^{*}$ we are guaranteed that $P_{j L}^{*}=P_{i R}$ and by the birthday paradox with a high probability there exist two plaintexts $P_{i}$ and $P_{j}^{*}$ such that $P_{j R}^{*}=P_{i L} \oplus \mathrm{K}_{L} \oplus$ ROL12 $\left(\mathrm{K}_{L}\right) \oplus F\left(P_{i R} \oplus \mathrm{K}_{R}, \mathrm{~K}_{L}\right)$. In such a pair the data is the same in both executions shifted by one round. It is easy to identify this pair, if it exists, by checking whether $C_{R}^{*}=C_{L}$. This test has a probability of $2^{-32}$ to pass accidentally, and thus only few pairs may pass this test.

A pair with this property relates the values of the two plaintexts and of the two ciphertexts to the key by equations 1 and 2 . Thus, such a pair reveals the value of

$$
F\left(P_{R} \oplus \mathrm{K}_{R}, \mathrm{~K}_{L}\right) \oplus F\left(C_{L} \oplus \mathrm{K}_{R}, \mathrm{~K}_{L}\right)=P_{R}^{*} \oplus P_{L} \oplus C_{L}^{*} \oplus C_{R}
$$

in which the only unknown value is $\mathrm{K}_{L} \oplus \mathrm{K}_{R}$. Out of the $2^{32}$ possible values of $\mathrm{K}_{L} \oplus \mathrm{K}_{R}$, only few values satisfy the equation. Using differential cryptanalytic[1,2] optimization techniques, such as difference distribution tables and storing the possible pairs of each of their entries in a special preprocessed table, the identification of the value of $K_{L} \oplus \mathrm{K}_{R}$ can be done in few 
operations. After we find $K_{L} \in K_{R}$, it is easy to calculate $K_{L} \fallingdotseq \operatorname{ROL12}\left(K_{L}\right)$ by equations I and 2 and to derive $K$ and $K^{*}$.

A similar chosen key known plaintext attack uses $2^{32}$ known plaintexts $P_{i}$ encrypted under an unknown key $\mathrm{K}$, and $2^{32}$ known plaintexts $P_{j}^{*}$ encrypted under the related key $\mathrm{K}^{*}=\left(\mathrm{K}_{R}, \operatorname{ROL} 12\left(\mathrm{~K}_{L}\right)\right)$. By the birthday paradox there is a high probability to have a pair in which the property holds. It is easy to identify this pair by the 32 common bits of the plaintexts and the 32 common bits of the ciphertexts. This pair may be used to reveal the keys in the same way as in the chosen key chosen plaintext attack.

\subsection{LOKI91}

The key scheduling algorithm of LOKI91 derives the subkeys of the even rounds in a different way than the subkeys of the odd rounds. In particular, the subkey of an even round $i$ is just a rotated value of the subkey of the previous round $i-1$, and it is independent of the value of round $i+1$. Thus, we cannot shift the subkeys and the data by one round in the second execution without changing their values. However, we can shift them by two rounds instead. In this case we define the second set of subkeys to be $\mathrm{K1}^{*}=\mathrm{K} 3, \mathrm{~K} 2^{*}=\mathrm{K} 4$ and so on. The keys themselves are $\mathrm{K}$ and $\mathrm{K}^{*}=\left(\mathrm{K}_{R}, \operatorname{ROL25}\left(K_{L}\right)\right)$. Since the shift is increased to two rounds, we cannot easily identify the pair we are looking for. Luckily, the two subkeys $\mathrm{K} 1$ and $\mathrm{K} 2$ share the same 32 bits, and thus given a plaintext $P$, we have only to guess 32 bits of the key in order to find the data before the third round, which we define as $P^{*}$.

In the chosen key chosen plaintext attack, the attacker chooses a random plaintext $P$. For each one of the $2^{32}$ possible values of $\mathrm{K} 1$ and $\mathrm{K} 2$, he calculates the data before the third round, and defines these $2^{32}$ values as $P_{i}^{*}$. Given the ciphertexts $C=\operatorname{LOKI} 91(P, K)$ and $C_{i}^{*}=\operatorname{LOKI} 91\left(P_{i}^{*}, K^{*}\right)$, he searches for the plaintext $P_{i}^{*}$ which was defined by the real values of $\mathrm{K} 1$ and $\mathrm{K} 2$ by verifying the relationships between the ciphertexts. The subkeys $\mathrm{K} 15^{*}$ and $\mathrm{K} 16^{*}$ share the bits of $\mathrm{K} 1$ and $\mathrm{K} 2$, which he has already guessed. Thus, it is easy to find the real values of $\mathrm{K} 1$ and $\mathrm{K} 2$ by calculating further from $C$ into $C_{i}^{*}$ by each possibility, and only the possibilities which result with the encrypted value of $C_{i}^{*}$ might have the real value. After we find these 32 bits of the keys, it is easy to find the other 32 bits by exhaustive search.

The best choice of a chosen key known plaintext attack uses $2^{16}$ plaintexts $P_{i}$ and $2^{48}$ plaintexts $P_{j}^{*}$. With high probability there is a pair $P_{i}$ and $P_{j}^{*}$. for which $P_{j}^{*}$ equals the data before the third round during the encryption of $P_{i}$. The attacker can identify the key by guessing the 32 bits of $\mathrm{K} 1$ and $\mathrm{K} 2$ and verifying which of which is possible in a way similar to the chosen key chosen plaintext attack.

\section{The Chosen plaintext attacks}

In this section we describe a chosen plaintext attack which reduces the complexity of exhaustive search using related keys. This attack can be combined with the attacks based on complementation properties[7] and its fastest variant is almost three times faster than the corresponding attacks based only on complementation properties. When this attack is used against 64 -bit blockciphers, it requires about $2^{32}-2^{37}$ chosen plaintexts, whose corresponding ciphertexts are to be stored in random access memory during the analysis.

The idea is similar to the attack based on the complementation property of DES[i]. The complementation property of DES suggests that whenever a plaintext $P$ is encrypted under a key $\mathrm{K}$ into a ciphertext $C=\operatorname{DES}(P, \mathrm{~K})$, then the complement of $P$ is encrypted by the complement of $\mathrm{K}$ into the complement of $C: \bar{C}=\operatorname{DES}(\bar{P}, \bar{K})$. The attack chooses a 


\begin{tabular}{rrr}
\hline Cycle Size & Number of Cycles & Number of Elements in the Cycles \\
\hline 1 & 16 & 16 \\
2 & 120 & 240 \\
4 & 16,320 & 65,280 \\
8 & $33,546,240$ & $268,369.920$ \\
Total & $33,562,696$ & $268.435,456^{\dagger}$ \\
\hline $268.435,456=2^{28}$ &
\end{tabular}

Table 1. Cycles of half-keys for LOKI89.

complementary pair of plaintexts $P_{1}$ and $P_{2}=\dot{P}_{1}$. Given their ciphertexts $C_{1}=\operatorname{DES}\left(P_{1}, \mathrm{~K}\right)$ and $C_{2}=\operatorname{DES}\left(P_{2}, \mathrm{~K}\right)$ under the same key $\mathrm{K}$, the attacker searches for the key $\mathrm{K}$ by trying all the keys $\mathrm{K}^{\prime}$ whose most significant bits are zero (i.e., half of the key space). For each such key, he encrypts $P_{1}$ into $C^{\prime}$ by $C^{\prime}=\operatorname{DES}\left(P_{1}, K^{\prime}\right)$. If $C^{\prime}=C_{1}$, it is very likely that $\mathrm{K}=\mathrm{K}^{\prime}$. In addition, the attacker can predict the ciphertext of $P_{1}$ under the key $\overline{\mathrm{K}}^{\prime}$ to be $\bar{C}_{2}$ without an additional encryption. If $C^{\prime}=\bar{C}_{2}$, it is very likely that $\mathrm{K}=\overline{\mathrm{K}}^{\prime}$, since due to the complementation property $\bar{C}_{2}=\operatorname{DES}\left(P_{1}, \overline{\mathrm{K}}\right)$. Otherwise, neither $\mathrm{K}^{\prime}$ nor $\overline{\mathrm{K}}^{\prime}$ can be the kev $K$. This attack can be carried out even under a known plaintext attack[1], given about $2^{33}$ known plaintexts, since it is very likely that two complementary plaintexts exist within $2^{33}$ random plaintexts due to the birthday paradox.

LOKI89 has several complementation properties[3,1,4,10,8]. A key complementation property causes any key to have 15 equivalent keys which encrypt any plaintext to the same ciphertext. These 15 keys are the original key XORed with the 15 possible 64-bit hexadecimal numbers whose digits are identical. Encryption with these keys results with the same inputs to the $F$ functions in all the 16 executions. Therefore, most of the keys are redundant and a known plaintext attack can be carried out with a complexity of $2^{60}$ rather than $2^{64}$.

Another complementation property of LOKI89 is due to the observation that XORing the key with an hexadecimal value gggggggghhhhhhhh and XORing the plaintext by iiiiiiiiiiiiiiii $_{x}$ where $g \in\left\{0_{x}, \ldots, F_{x}\right\}, h \in\left\{0_{x}, \ldots, F_{x}\right\}$ and $i=g \oplus h$ results in XORing the ciphertext by iiiiziiiiiiiiiii ${ }_{x}$. This property can be used to reduce the complexity of a chosen plaintext attack by a further factor of 16 to $2^{56}$.

The attack we present in this section can predict the values of additional ciphertexts generated from additional plaintexts under related keys. Let $P$ be any plaintext, $\mathrm{K}$ be any key and $C$ be the ciphertext $C=\operatorname{LOKI} 89(P, \mathrm{~K})$. Let $\mathrm{K}^{*}=\left(\mathrm{K}_{R}, \operatorname{ROL12}\left(\mathrm{K}_{L}\right)\right)$ and let $P^{*}$ be the plaintext whose data before the first round of the encryption under $K^{*}$ is the same as the data before the second round during the original encryption of $P$ under $\mathrm{K}$. Then, the first 15 rounds of the encryption $C^{*}=\operatorname{LOKI89}\left(P^{*}, \mathrm{~K}^{*}\right)$ have exactly the same data and subkeys as the last 15 rounds of the original encryption of $P$, and the right half of $C^{*}$ equals the left half of $C$ (i.e., $C_{R}^{*}=C_{L}$ ).

For each key, there is one equivalent key whose four most significant bits are zero, and one complement key whose four most significant bits of its both halves are zero. In the following definition, the next operation rotates an half-key by 12 bits (as is done in the key scheduling algorithm every round) and finds the supposed equivalent value of the result.

Definition 1 The next operation takes a 32-bit value, rotates it 12 bits to the left (ROL12) and XORs it with an 32-bit hexadecimal number whose all digits are equal. such that the four most significant bits of the result are zero.

Table 1 shows the cycle size, number of cycles and the total number of elements in the cycles 
generated by the next operation. We see that almost all the cycles have the maximal size eight.

In our attack we use the observation that the shift of one round of the data and the subkeys can be done in both the backward and forward directions. Therefore. each trial key can predict ciphertexts under three related keys, and thus the attack requires to try about a third of the number of kevs required by the attack based on the complementation properties. Usually it is not possible to find a subset of the keys which satisfy the related keys conditions and contain exactly a third of all the keys. The best choice for LOKI89 is to try $3 / 8$ of the keys. We preprocess a list of half-keys $\left\{L_{i}\right\}$, with the properties: (1) The four most significant bits of all the values in the list are zero, and (2) The list contains exactly one value from each cycle of the next operation. This list contains about $2^{25}$ half-keys. The list can either be stored in memory using about $4 \cdot 2^{25}=2^{27}$ bytes, or stored as a bitmap using $2^{28} / 8=2^{25}$ bytes ${ }^{2}$.

The attack requires $2^{37}$ chosen plaintexts whose ciphertexts are stored in a random access memory (whose size is $2^{40}$ bytes). The attack is as follows:

1. Choose any plaintext $P_{0}$, and calculate the 15 plaintexts $P_{i}, i \in\left\{1_{x}, \ldots, F_{x}\right\}$, by $P_{i}=P_{0} \oplus$ iiiiiiiiiziziiii $_{x}$.

2. For each plaintext $P_{i}$, choose the additional $2^{32}$ plaintexts $P_{i, k}=\left(P_{i R}, P_{i L} \doteq k\right)$ whose left halves are the right half of $P_{i}$ and whose right halves receive all the possible values by XORing all the possible 32-bit values $k$ to the left half of $P_{i}$.

3. For each plaintext $P_{i}$, choose the additional $2^{32}$ plaintexts $P_{i, k}^{*}=\left(P_{i R} \oplus k, P_{i L}\right)$ whose right halves are the left half of $P_{i}$ and whose left halves receive all the possible values by XORing all the possible 32-bit values $k$ to the right half of $P_{i}$.

4. Given the ciphertexts $\left\{C_{i}\right\},\left\{C_{i, k}\right\},\left\{C_{i, k}^{*}\right\}$, try for each pair of half-keys $\left(L_{i}, L_{j}\right)$ all the 24 keys $\mathrm{K}^{\prime}$ of the form $\mathrm{K}^{\prime}=\left(\operatorname{RORm}\left(L_{i}\right), \operatorname{ROL} n\left(L_{j}\right)\right)$, where $\mathrm{m}$ is a multiple of four and $n$ is either $n=m, n=m+4$, or $n=m+8$. Figure 5 shows the choice of such 24 trial keys, which are denoted by $\dagger$. All the keys are covered by the trial keys. The trial keys $K^{\prime}$ are covered by themselves. The other keys are covered by the key relation property by trial keys created from $\left(L_{j}, L_{i}\right)$. These keys are surrounded together with the swapped value of their trial keys. Examples of four such triples are marked in gray. The keys denoted by * are covered by two trial keys. The figure should be interpreted cyclically through its edges. This is the best coverage possible for LOKI89.

5. Encrypt the plaintext $P_{0}$ under each trial key $\mathrm{K}^{\prime}$ into $C^{\prime}=\operatorname{LOKI} 89\left(P_{0}, \mathrm{~K}^{\prime}\right)$.

6. If $C^{\prime}$ equals one of the values $C_{i} \oplus$ iiiiiiiiiiiiiii $_{x}$, the original key is likely to be either $K=K^{\prime} \oplus 00000000$ iiiiiiii $_{x}$ or any one of its 15 equivalent keys.

$i$. Fix $k$ to be the output of the $F$ function in the first round of the encryption of $P_{0}$ under the key $\mathrm{K}^{\prime \prime}$. If $C_{L}^{\prime}$ equals one of the 16 values $C_{i, k R} \oplus i i_{i i i i i i_{x}}$, continue encryption of $P_{0}$ with a seventeenth round (just calculate one additional round from $C^{\prime}$ using the subkey $\mathrm{K} 7^{\prime}$ which can be easily derived from the key $\mathrm{K}^{\prime}$ ), and if the result $C^{\prime \prime}$ equals $C_{i, k} \oplus i i i i i i i i i i i z i z i_{x}$, then the original key is likely to be $\mathrm{K}=\left(\mathrm{K}_{R}^{\prime}, \operatorname{ROL12}\left(\mathrm{K}_{L}^{\prime}\right) \oplus i i i i i i_{x}\right)$ or any one of its 15 equivalent keys.

8. Calculate one additional round backwards from $P_{0}$ using the subkey $\mathrm{KO}^{\prime}$ which can be easily derived from the key $\mathrm{K}^{\prime}$, and fix $k$ to be the output of the $F$ function in this round. If the data after the fifteenth round during the encryption of $P_{0}$ under the key

\footnotetext{
${ }^{2}$ We have also devised an additional algorithm which chooses the trial keys on the fly and does not require a list of potential half-keys. However, due to technical detajls, the efficiency of the attack is reduced. Since the memory space required by the attack is much larger than the list of potential half-keys, this list does not affect the space complexity of the attack.
} 


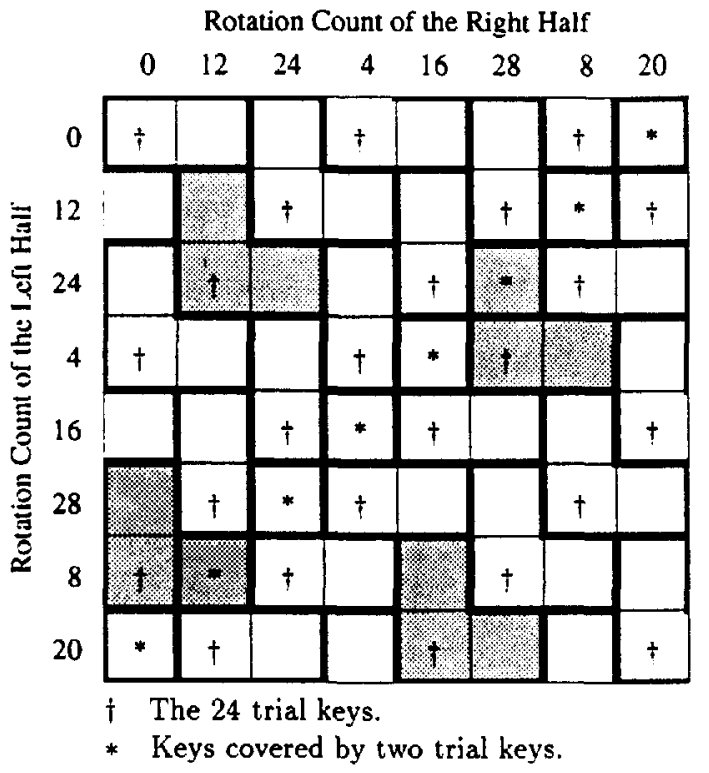

Figure 5. The choice of the trial keys.

$\mathrm{K}^{\prime}$ equals one of the 16 values $C_{i, k} \oplus$ iiiiiiiiiiiiiii $_{x}$ (for some $i$ ), the original key is likely to be $K=\left(\operatorname{ROR} 12\left(K_{R}^{\prime}\right), K_{L}^{\prime} \oplus\right.$ iiiiiiii $\left._{z}\right)$ or any one of its 15 equivalent keys ${ }^{3}$.

The encryption key $\mathrm{K}$ must be recognized by either step 6 , step $\bar{\tau}$. or step 8 . Since the comparisons in steps 6,7 and 8 are much faster than a trial encryption, the complexity of the attack is the total complexity of step 5 . If the number of elements in each cycle would have been divisible by three, the complexity of the attack would decrease relatively to the attack based on complementation properties by a factor of $1 / 3$. In the case of LOKI89 this factor is only $3 / 8$, and the complexity of the attack is $(3 / 8) \cdot\left(2^{28}\right)^{2}=1.5 \cdot 2^{54}$ trial encryptions 4 .

The application of this attack to LOKI91 is similar. The main two differences are: (1) The plaintexts are chosen with respect to changes in the half of the key which affects the first two rounds, rather than changing the plaintext's halves directly. (2) The trial keys generated in step 4 of the attack cover only half of the keys, since there is only one complementation property. Thus, we use twice as many trial keys, namely the original trial keys and the same trial keys whose right halves are complemented. The complexity of this attack on LOKI91 is $1.375 \cdot 2^{61}$, which is about 5.8 times faster than exhaustive search.

\footnotetext{
'This part of the algorithm requires the calculation of one additional round for any trial key $\mathbf{K}^{\prime}$, which slows the attack by a factor of $17 / 16$. In parallel hardware implementations, this behavior can be solved easily. In software, it can also be solved by using large hash tables and checking the existence of ciphertexts before the calculation of the additional round.

${ }^{4}$ If we choose carefully the order of trying the trial keys, we can reduce the average case complexity slightly from $1.5 \cdot 2^{53}$ by about $8 \%$.
} 


\begin{tabular}{lrrrrrrrrrrrrrrrr}
\hline Round & 1 & 2 & 3 & 4 & 5 & 6 & 7 & 5 & 9 & 10 & 11 & 12 & 13 & 14 & 15 & 16 \\
Shifts & 1 & 1 & 2 & 2 & 2 & 2 & 2 & 2 & 1 & 2 & 2 & 2 & 2 & 2 & 2 & 1 \\
Modified variant & 2 & 2 & 2 & 2 & 2 & 2 & 2 & 2 & 2 & 2 & 2 & 2 & 2 & 2 & 2 & 2 \\
\hline
\end{tabular}

Table 2. The numbers of shifts in the key scheduling algorithm of DES. and a modified variant vulnerable to the related keys attacks.

\section{Application to Lucifer}

In Lucifer[16]. the subkeys are derived by shifting the key by a fixed number of bits every round and selecting specific bits as the subkeys. and thus the derivation algorithm of the subkeys from the previous subkeys is the same in all the rounds. Therefore. chosen key attacks similar to the attacks on LOKI89 are applicable, but since the blocksize and the size of the key are 128 bits, the complexities become $2^{33}$ for the chosen key chosen plaintext attack and $2^{65}$ for the chosen key known plaintext attack. Lucifer has no complementation properties, and thus the complexity of the chosen plaintext attack is $1.5 \cdot 2^{126}$.

\section{DES}

DES[14] is not vulnerable to the related keys attacks since the number of shifts in the key scheduling algorithm is not the same in all the rounds. While usually the key registers are shifted by two bits after each round, they are shifted only by one bit after the first. the ninth and the fifteenth rounds. However. if we modify this shift pattern to shift the key registers by the same number of bits in all the rounds (either one or two or any other number including seven which was suggested in [6]), the resultant cryptosystem becomes vulnerable to the related keys attacks. Table 2 describes the numbers of shifts before each round in the key scheduling algorithm of DES and a variant with modified shift numbers which is rulnerable to the related keys attacks.

\section{Summary}

In this paper we described new cryptanalytic attacks which are applicable to the LOKI family of blockciphers and to Lucifer. These new attacks are based on the structure of the key scheduling algorithms. These attacks are independent of the number of the rounds of the cipher. The same attacks could be applicable to DES if only minor changes would be made to the shift pattern of its key scheduling algorithm, and thus these attacks show how so small points in the design of a cipher can contribute to its strength. The results of the related keys attacks are summarized in table 3 .

\section{References}

[1] Eli Biham. Adi Shamir. Differential Cryptanalysis of the Data Encryption Standard. Springer-Verlag. 1993.

[2] Eli Biham. Adi Shamir. Differential Cryptanalysis of DES-like Cryptosystems. Journal of Cryptology; Vol. 4. No. 1. pp. 3-72. 1991. 


\begin{tabular}{|c|c|c|c|c|}
\hline & LOKI89 & LOKI91 & Lucifer & Modified DES" \\
\hline \multicolumn{5}{|l|}{ Chosen Plaintext: Related Keys } \\
\hline Complexity of Attack & $1.5 \cdot 2^{54}$ & $1.375 \cdot 2^{61}$ & $1.5 \cdot 2^{126}$ & $1.43 \cdot 2^{53}$ \\
\hline Chosen Plaintexts Required & $2^{37}$ & $2^{34}$ & $2^{65}$ & $2^{34}$ \\
\hline \multicolumn{5}{|l|}{ Chosen Key } \\
\hline Chosen Plaintexts & $2^{17}$ & $2^{32}$ & $2^{33}$ & $2^{17}$ \\
\hline Known Plaintexts & $2^{33}$ & $2^{48}$ & $2^{65}$ & $2^{33}$ \\
\hline \multicolumn{5}{|l|}{ Previously known attacks } \\
\hline Differential Cryptanalysis & - & - & - & $2^{47}$ \\
\hline Complementation Properties ${ }^{\dagger}$ & $2^{56}$ & $2^{63}$ & - & $2^{55}$ \\
\hline Exhaustive Search & $2^{60}$ & $2^{64}$ & $2^{128}$ & $2^{56}$ \\
\hline
\end{tabular}

- The shift pattern of the key registers in the key scheduling algorithm is modified to have the same number of shifts in all the rounds (see Table 2).

$\dagger$ Either two chosen plaintexts required, or $2^{33}$ known plaintexts. For LOKI89,

16 chosen plaintexts are required to achieve this complexity.

: One known plaintext required.

Table 3. Results of the related keys attacks.

[3] Eli Biham. Adi Shamir, Differential Cryptanalysis of Snefru, Khafre, REDOC-II. LOKI and Lucifer (extended abstract), Lecture Notes in Computer Science. Advances in Cryptology. proceedings of CRYPTO'91, pp. 156-171, 1991.

[1] Lawrence Brown, Matthew Kwan, Josef Pieprzyk, Jennifer Seberry, Improving Resistance to Differential Cryptanalysis and the Redesign of LOKI, Lecture Notes in Computer Science, Advances in Cryptology, proceedings of ASIACRYPT'91, to appear.

[5] Lawrence Brown, Josef Pieprzyk, Jennifer Seberry, LOKI - A Cryptographic Primitive for Authentication and Secrecy Applications, Lecture Notes in Computer Science, Advances in Cryptology, proceedings of AUSCRYPT'90, pp. 229-236, 1990.

[6] Lawrence Brown. Jennifer Seberry, Key Scheduling in DES Type Cryptosystems. Lecture Totes in Computer Science. Advances in Cryptology, proceedings of AUSCRYPT'90, pp. 221-228. 1990.

[i] M. E. Hellman, R. Merkle, R. Schroppel, L. Washington, W. Diffie. S. Pohlig and P. Schweitzer, Results of an Initial Attempt to Cryptanalyze the NBS Data Encryption Standard, Stanford university, September 1976.

[3] Lars Ramkilde Knudsen, Crypanalysis of LOKI, Lecture Notes in Computer Science, Advances in Cryptology, proceedings of ASIACRYPT'91, to appear.

[9] Lars Ramkilde Knudsen, Crypanalysis of LOKI91, Lecture Notes in Computer Science, Advances in Cryptology, proceedings of AUSCRYPT'92, to appear.

[10] Matthew Iiwan. Josef Pieprzyk, A General Purpose Technique for Locating liey Schtduling Wtakness in DES-Like Cryptosystems, Lecture Notes in Computer Science. Advances in Cryptology, proceedings of ASIACRYPT'91, to appear.

(11) Xuejia Lai. James L. Massey, Sean Murphy, Markov Ciphers and Differential Cryptanalysis. Lecture Notes in Computer Science, Advances in Cryptology, proceedings of ELROCRYPT'91.pp. 17-38, 1991.

[12] Xuejia Lai. On the Design and Security of Block Ciphers, Ph.D. thesis. Swiss Federal Institue of Technology, Zurich. 1992. 
[13] Shoji Miyaguchi. Akira Shiraishi, Akihiro Shimizu, Fast Data Encryption Algorithm FEA $L-8$, Review of electrical communications laboratories. Vol. 36. No. 4. pp. 433-437, 1988 .

[14] National Bureau of Standards, Date Encryption Standard, U.S. Department of Commerce, FIPS pub. 46, January 1977.

[15] Akihiro Shimizu, Shoji Miyaguchi, Fast Data Encryption Algorithm FEAL, Lecture Notes in Computer Science, Advances in Cryptology, proceedings of EUROCRYPT'87, pp. 267-278, 1987.

[16] Arthur Sorkin. Lucifer, a Cryptographic Algorithm, Cryptologia, Vol. 8, No. 1. pp, 22-41, January 1984. 